\title{
Editorial
}

\section{Qualitative Analysis of Differential Equations}

\author{
Ondřej Došlý, ${ }^{1}$ Jaroslav Jaroš, ${ }^{2}$ Mervan Pašić, ${ }^{3}$ and Norio Yoshida ${ }^{4}$ \\ ${ }^{1}$ Department of Mathematics and Statistics, Masaryk University, 61137 Brno, Czech Republic \\ ${ }^{2}$ Department of Mathematical Analysis and Numerical Mathematics, Comenius University, 84248 Bratislava, Slovakia \\ ${ }^{3}$ Department of Mathematics, University of Zagreb, 10000 Zagreb, Croatia \\ ${ }^{4}$ Department of Mathematics, University of Toyama, Toyama 930-8555, Japan
}

Correspondence should be addressed to Norio Yoshida; nori@sci.u-toyama.ac.jp

Received 1 August 2013; Accepted 1 August 2013

Copyright (C) 2013 Ondřej Došlý et al. This is an open access article distributed under the Creative Commons Attribution License, which permits unrestricted use, distribution, and reproduction in any medium, provided the original work is properly cited.

It is our pleasure to provide this special issue on qualitative analysis of differential equations in International Journal of Differential Equations.

Qualitative analysis has proved to be an important and useful tool to investigate the properties of solutions of differential equations, because it enables to analyze differential equations without solving analytically and numerically. The study of qualitative properties of differential equations has a long history, and qualitative theories have been developed for various equations such as ordinary differential equations, functional differential equations, abstract differential equations, dynamical systems, integral equations, difference equations, partial differential equations, functional partial differential equations and so forth. Since the qualitative analysis of differential equations is related to both pure and applied mathematics, its applications to various fields such as science, engineering, and ecology have been extensively developed.

This special issue contains papers which treat a number of important and attractive problems related to existence of positive periodic solutions for periodic neutral LotkaVolterra system with distributed delays and impulses, dynamics of a Gross-Pitaevskii equation with phenomenological damping, characterization for rectifiable and nonrectifiable attractivity of nonautonomous systems of linear differential equations, positive periodic solutions of cooperative systems with delays and feedback controls, oscillations of a class of forced second-order differential equations with possible discontinuous coefficients, analysis of mixed elliptic and parabolic boundary layers with corners, unboundedness of solutions of Timoshenko beam equations with damping and forcing terms, and fractal oscillations of chirp functions and applications to second-order linear differential equations.

The paper by Z. Luo and L. Luo discusses the existence of positive periodic solutions for a class of impulsive neutral Lotka-Volterra system with distributed delays by using a fixed point theorem of strict-set contraction. Some criteria that guarantee the existence of at least one positive periodic solution of the system are established.

The research article of $\mathrm{R}$. Colucci et al. deals with the dynamical behavior of solutions of an $n$-dimensional nonlinear Schrödinger equation with potential and linear derivative terms under the presence of phenomenological damping. The authors of that paper obtain conditions for the existence of a compact global attractor and find bounds for its Hausdorff and fractal dimensions.

In the paper by Y. Naito and M. Pašić, attractivity of all solutions near zero as time variable goes to zero of a class of nonautonomous linear differential system is studied by a kind of singular behaviour of eigenvalues of a corresponding matrix system. Moreover, a necessary and sufficient condition for a new notion, the so-called nonrectifiable attractive solutions (solution's curves of infinite length), is derived by an additional singular condition on eigenvalues of the matrix system. These results are based on some new lemmas on the rectification of planar curves near a point.

T. Niyaz and A. Muhammadhaji study a class of periodic $n$ species cooperative Lotka-Volterra systems with continuous time delays and feedback controls. Based on the continuation theorem of the coincidence degree theory developed by 
Gaines and Mawhin, some new sufficient conditions on the existence of positive periodic solutions are established.

The paper authored by S. Miličić et al. enlarges some recently published results on the classic oscillation of secondorder forced differential equations to some related equations that allow discontinues coefficients. In such a class of differential equations, instead of the classic $C^{2}$-solutions, the first derivatives of solutions are absolutely continuous functions. It causes some difficulties in using the Riccati transformation. It is resolved by a new pointwise comparison principle for ordinary differential inequalities with locally Lipschitz nonlinear term and bounded measurable coefficients.

The work of G.-M. Gie et al. addresses the asymptotic behavior at small diffusivity of solutions to a convectiondiffusion equation in a rectangular domain. The validity of their proposed asymptotic expansions in suitable Sobolev spaces is proved via the energy estimates rather than the maximum principle.

In the paper by K. Kobayashi and N. Yoshida, unboundedness results for Timoshenko beam equations with damping and forcing terms are presented. Three kinds of end conditions are considered, and it is shown that the magnitude of the displacement of the beam grows up to infinity as time passes under some assumptions on the forcing term.

The paper of M. Pašić and S. Tanaka deals with a new lemma for calculating the fractal (box-counting) dimension of graph of any real continuous function. It is illustrated to a general form of chirp functions which oscillates near a finite point. As a consequence, fractal oscillations near a point are derived for some classes of second-order linear differential equations generated by chirp functions.

\section{Acknowledgment}

The guest editors of this special issue would like to express their sincere gratitude to all authors for sending their new papers for publication. Thanks are also due to the reviewers, whose professional comments and valuable suggestions guaranteed the high quality of these selected papers. We hope that this special issue will be useful and fruitful for researchers working in qualitative analysis of differential equations and related areas and will help stimulate further progress in this important branch of differential equations.

Ondřej Došlý

Jaroslav Jaroš

Mervan Pašić

Norio Yoshida 


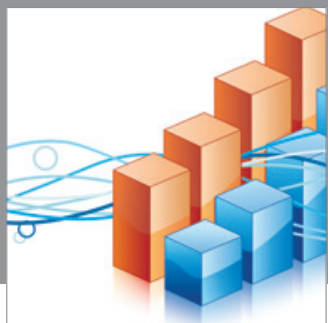

Advances in

Operations Research

mansans

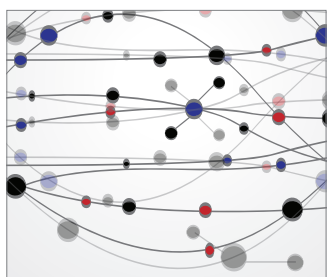

The Scientific World Journal
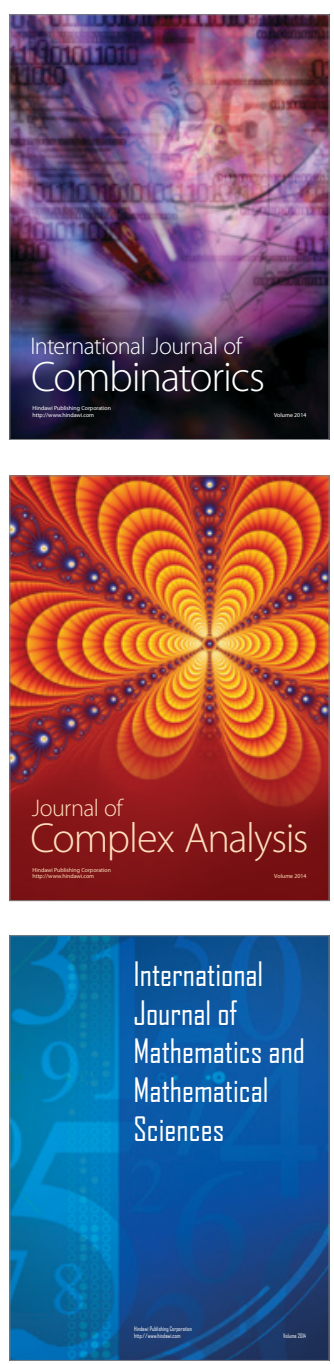
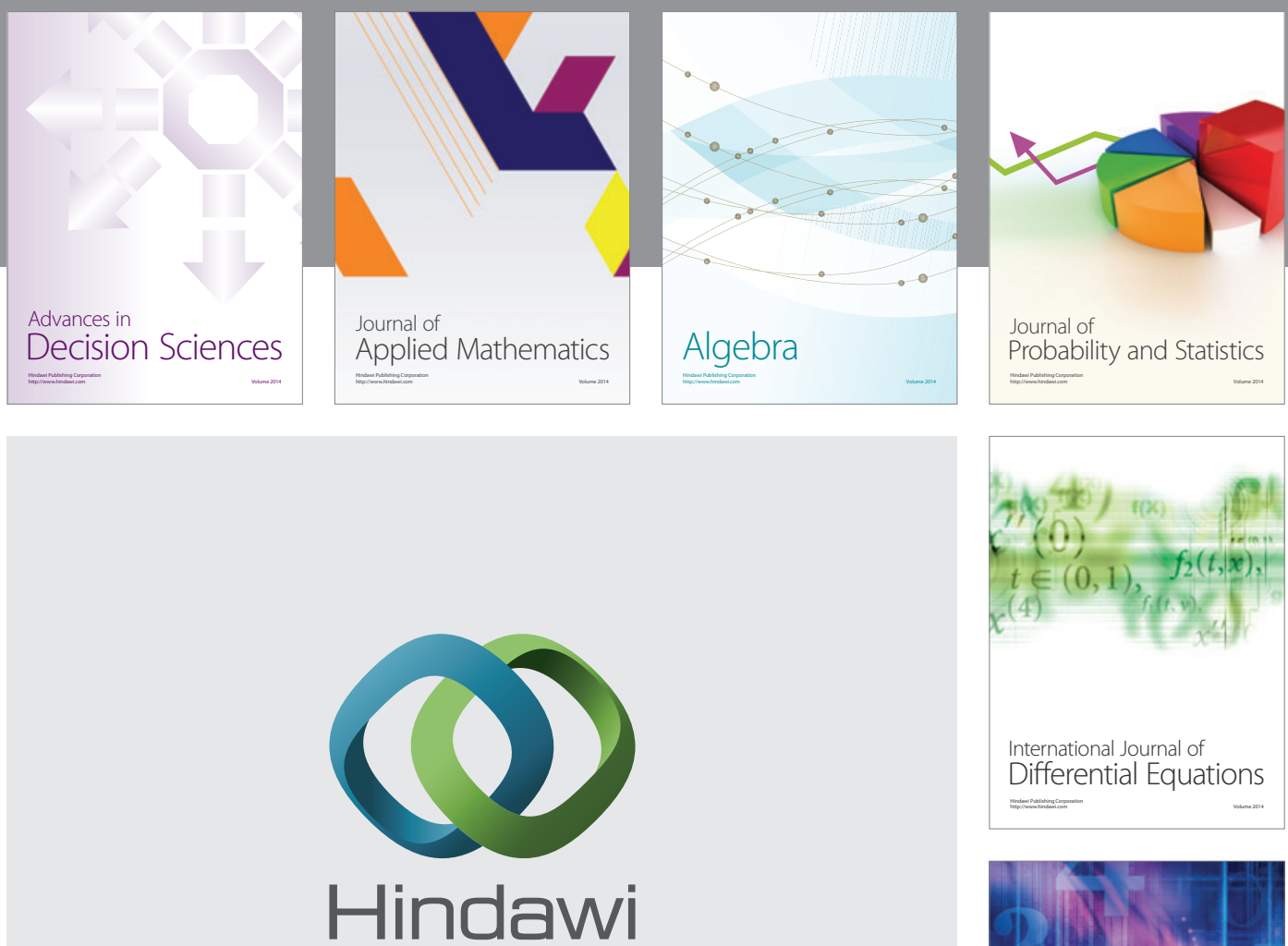

Submit your manuscripts at http://www.hindawi.com
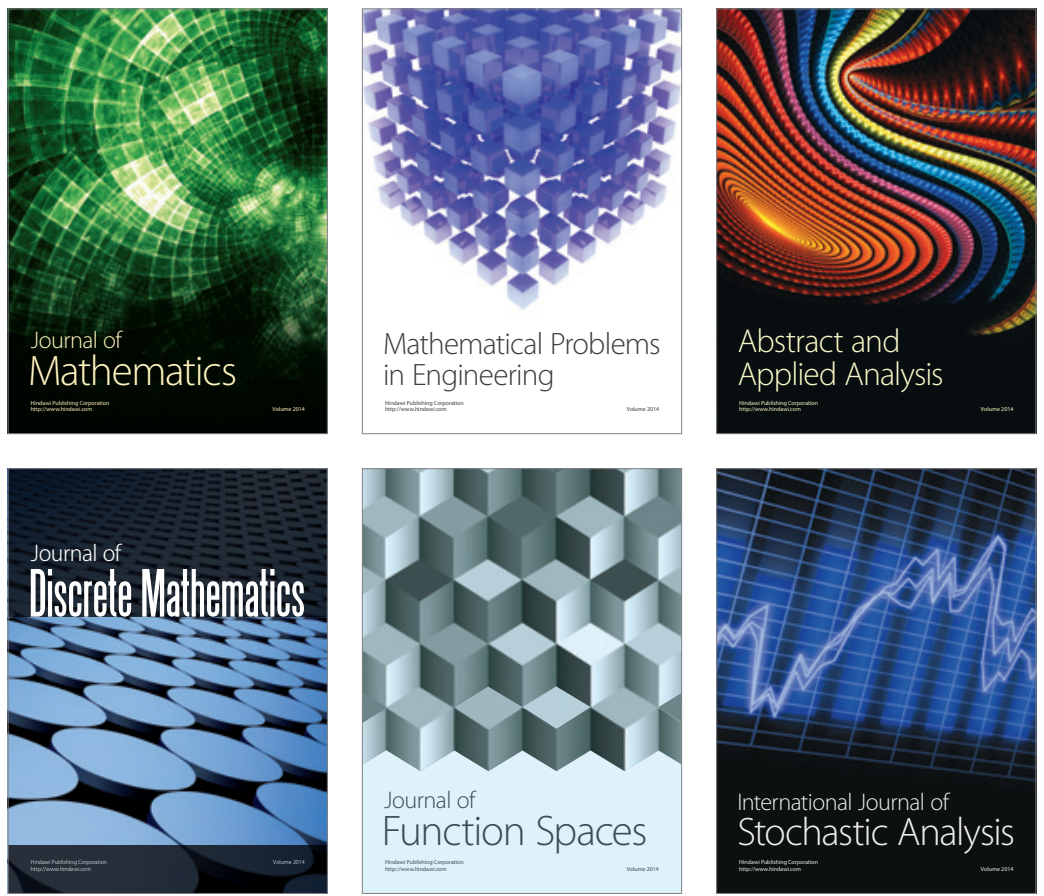

Journal of

Function Spaces

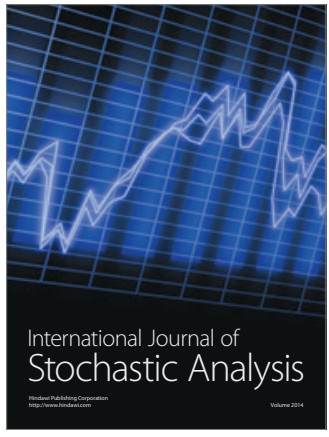

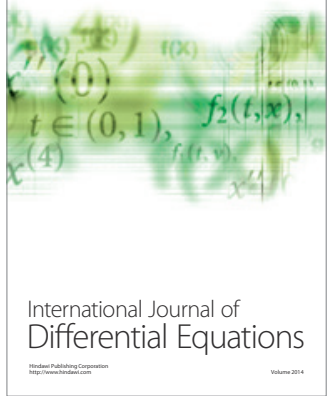
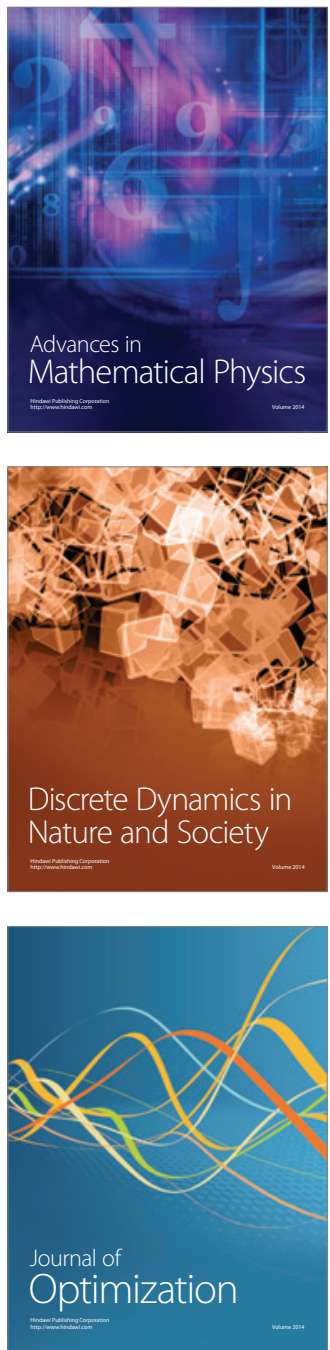